Venkatesh et al., Afr J Tradit Complement Altern Med., (2017) 14 (3): 167-174

doi:10.21010/ajtcam.v14i3.18

PURIFICATION AND FRACTIONAL ANALYSIS OF METHANOLIC EXTRACT OF WEDELIA TRILOBATA POSSESSING APOPTOTIC AND ANTI-LEUKEMIC ACTIVITY.

\author{
Uday Venkatesh $^{1}$, Chethan Javarasetty ${ }^{2}$ and Satish Kumar Murari ${ }^{3 * *}$ \\ ${ }^{1}$ Department of Biochemistry, Bharathiar University, Coimbatore-641 046, Tamilnadu, India. \\ ${ }^{2}$ Central Coffee Research Institute, Balehonoor, Chikkamagalur-577 117, Karnataka, India. \\ ${ }^{3}$ Department of Chemistry, P.E.S. College of Engineering, Mandya-571 401, Karnataka, India.
}

*Corresponding author Email: pesmurari@gmail.com

\begin{abstract}
:
Background: Wedelia trilobata (L.) Hitch (WT), commonly known as yellow dots or creeping daisy, is a shrub possessing potent biological activities, and is traditionally used a medicinal plant in Ayurveda, Siddha and Unani systems of medicines, and it has also been tried against leukemia cell line MEG- 01. In the present study, purification and screening of the plant was done for bioactive compounds in methanolic extract of WT for apoptotic and antileukemia activity.

Materials and methods: The methanolic extract of WT was initially purified through thin layer chromatography (TLC) and screened for the apoptotic and anti-leukemia activities. The positive band of TLC was subjected to silica gel column chromatography for further purification and the fractions obtained from it were screened again for antileukemia activity through thymidine uptake assay and apoptotic activity by DNA fragmentation, nuclear staining and flow cytometry assays. The fraction with positive result was subjected to HPLC for analysis of bioactive components.

Results: Out of many combinations of solvents, the methanol and dichloromethane combination in the ratio 6:4 has revealed two bands in TLC, among which the second band showed positive results for apoptotic and anti-leukemic activities. Further purification of second band through silica gel chromatography gave five fractions in which the $3^{\text {rd }}$ fraction gave positive results and it shows single peak during compositional analysis through HPLC.

Conclusion: The single peak revealed through HPLC indicates the presence of pure compound with apoptotic and antileukemia activities encouraging for further structural analysis.
\end{abstract}

Key words: Wedelia trilobata, MEG-01, Antiproliferation, Apoptosis.

\title{
Introduction:
}

Approach towards natural products, like plant extracts, for healthcare needs is increasing consistently due to the side-effects of chemically synthesized drugs and the decrease in the potency of these drugs for effective treatment is due to the development of resistance by various pathogens. As per WHO, more than $80 \%$ of the world`s population depends on traditional medicine. Though traditional medicine being less potent in its action compared to chemically synthesized drugs, it has many other advantages, such as: minimal side-effects and maintaining being effective for the disease. Bioactive compounds from plant extracts provide unmatched chemically diverse compositions possessing the biological activity such as anti-microbial, anti-oxidants, anti-cancer, analgesic etc., which can be used to treat infectious as well as chronic diseases (Veeramuthu et al. 2006). Certain plant extracts like diterpenes possessing basic biological activity are also used as structural building block to synthesize bioactive compounds (Barrero et al. 2012; Barrero et al. 2006). Relying on the plant extracts for drug discovery and designing is a neve rending process which also gives maximum possibility for combinatorial treatment with other drugs to increase the effectiveness.

Leukemia, one of the major diseases in the world, has a statistical record of 1,450 deaths in United States during 2015 and overall survival rate of adults with ALC is very less (American Cancer Society. 2015). Though advanced treatment strategies were being developed against leukemia in parallel drug designing through traditional method via plant extracts place an important role to create wide range of cost effective treatment.

Asteraceae family plants are well known for its biological activities like antimicrobial, antifungal, antioxidant (Casiglia et al. 2016; $\mathrm{Na}$ An et al. 2016) and anticancer activity. Methanolic extract of Wedelia trilobata (Asteraceae family) has shown anti-leukemia activity (Uday et al. 2016), fortified by the previous results, here we have purified and screened further for the anti-leukemic activity. Chromatographic techniques like TLC and HPLC was employed for the stepwise screening of positive fractions and studied for their anti-leukemia and apoptotic assays using MEG01 cell lines. 
Venkatesh et al., Afr J Tradit Complement Altern Med., (2017) 14 (3): 167-174 doi:10.21010/ajtcam.v14i3.18

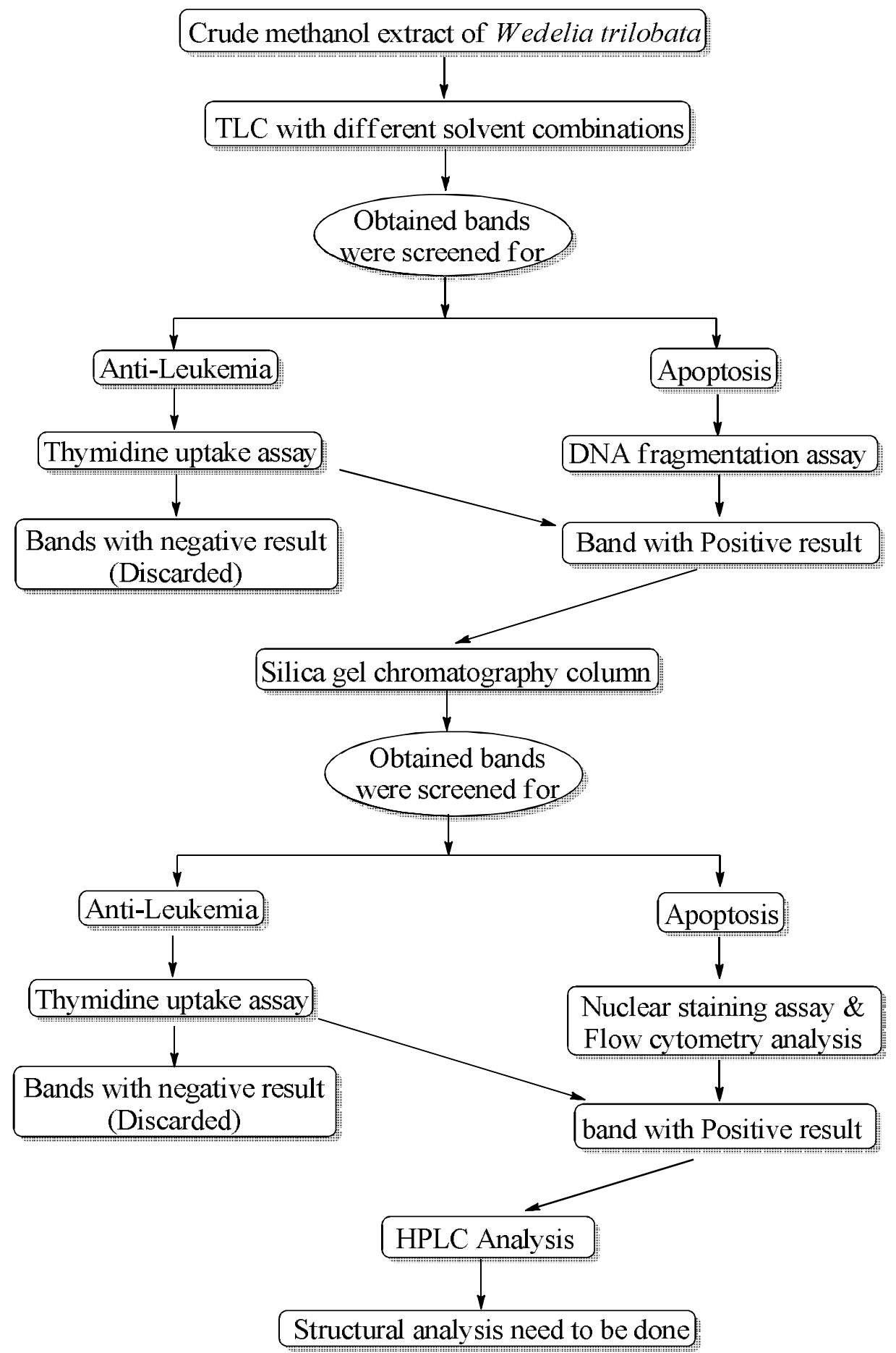

Figure 1: A brief explanation of the entire process done in the study

Materials and methods:

Collection of plant and obtaining crude extracts of Wedelia trilobata:

The characteristic features for identification of plant and its habitat and also procedure for the preparation of plant extracts of Wedelia trilobata has been followed as explained earlier (Uday et al. 2016). 
Venkatesh et al., Afr J Tradit Complement Altern Med., (2017) 14 (3): 167-174 doi:10.21010/ajtcam.v14i3.18

\section{Thin layer chromatography (TLC)}

This is the rapid and inexpensive method for the identification of number of compounds present in a crude sample and for the separation of plant extracts into different fractions (Sasidharan et al. 2011). The TLC plates of size $15 \mathrm{~cm}$ in length and $8 \mathrm{~cm}$ in width along with $0.5 \mathrm{~mm}$ thickness (E. Merck, Germany) were used. The extract was spotted on the plate and solvent front was made to run up to $10 \mathrm{~cm}$. Then the plates were dried and visualized under UV light. The two combination systems of solvents with different concentration ratio were used to separate the compositional mixtures in methanolic extract of the plant as mentioned below. The bands obtained were scraped from the plate and dissolved in methanol. The mixture was then centrifuged to separate from silica and the obtained supernatant was further used for antiproliferation and apoptotic assays.

Table 1: TLC done with the mentioned solvent combinations at different ratios with the results as noted.

\begin{tabular}{|l|l|}
\hline $\begin{array}{l}\text { Solvent combination taken in the ratio } \\
\text { of 1:9 to 9:1 }\end{array}$ & Observation \\
\hline Methanol : hexane & No separation \\
\hline Methanol: petroleum ether & Smear formation \\
\hline Chloroform : hexane & No separation \\
\hline Chloroform: pet. ether & No separation \\
\hline Methanol : dichloromethane & At the ratio of 6:4, two bands were obtained \\
\hline
\end{tabular}

Activity analysis of the fractions obtained from TLC:

Cell line culture and Thymidine uptake assay: $5 \times 10^{4}$ MEG-01 human Megakaryoblastic cells (Sigma Aldrich, India) were seeded per well and cultured in RPMI 1640 (Sigma Aldrich) supplemented with 10\% Fetal Bovine Serum (FBS) and $5 \% \mathrm{CO}_{2}$ at $37^{\circ} \mathrm{C}$ for $24 \mathrm{~h}$. When the cells becomes $80 \%$ confluent, $1 \mu \mathrm{l}(\mu \mathrm{Ci} / \mathrm{ml})$ of $[3 \mathrm{H}]$ Thymidine was added and followed by the addition of TLC separated samples in a quantity of $2 \mu \mathrm{l}, 4 \mu \mathrm{l}$ and $8 \mu \mathrm{l}$ respectively. After $48 \mathrm{~h}$ of incubation, the media was removed and cells were processed to measure the radioactivity using liquid scintillation counter. The proliferation rate was correlated through graphical method (Giridharan et al. 2002; Belakavadi et al. 2005).

DNA fragmentation assay: The MEG01 cells treated with TLC fractions and the untreated cells were subjected to DNA isolation by incubation with lysis buffer for $1 \mathrm{~h}$ at room temperature. The lysate was centrifuged to separate the contaminants, then the DNA was precipitated using phenol: chloroform: isoamyl alcohol mixture. The precipitated DNA sample was pooled out and dried through vacuum evaporator. The obtained DNA samples were assessed electrophoretically in 1\% agarose gel (Park et al. 2013).

Silica gel chromatography (SGC): The bulk quantity of the positive fraction was obtained through preparative TLC method and subjected to further purification using silica gel (Merk) column chromatography (Fatma et al. 2016). The minimum quantity of sample was dissolved in the solvent mixture of methanol and dichloromethane at 6:4 ratio and loaded to the silica packed column. The five different fractions separated (confirmed through TLC) were collected and used for the study of activity.

\section{Activity analysis of the fractions obtained from Silica gel chromatography:}

Thymidine uptake assay: The five fractions obtained from column chromatography were analyzed for the antiproliferation activity through thymidine uptake assay and the procedure was followed as explained in earlier methods (Giridharan et al. 2002; Belakavadi et al. 2005).

Nuclear staining: The apoptotic activity of the five different column chromatography fractions was analyzed through nuclear staining by (EtBr) Ethidium Bromide and Acridine orange solutions. The MEG-01 cells treated with the SGC obtained fractions and the untreated cells were washed in ice-cold Phosphate buffer saline (PBS) and incubated with $3.7 \%$ paraformaldehyde for $10 \mathrm{~min}$ at room temperature for fixation of cells. The cells were washed again with PBS and 4',6-diamidino-2-phenylindole (DAPI) solution was added to stain for $10 \mathrm{~min}$ at room temperature and cells were rewashed with PBS and the fluorescent cell nuclei were analyzed through microscopy as reported earlier (Srinivas et al, 2003).

Flow cytometry analysis: The induction of apoptosis in MEG-01 cells by the sample (column purified third fraction) was analyzed using BD Annexin V: FITC Apoptosis Detection Kit I (BD bioscience). The principle behind the study was to analyze the translocated phosphatidylserine from inside to outer plasma membrane of the apoptotic cell which is not present in the normal cells. Fluorochrome tagged Annexin $\mathrm{V} \mathrm{a} \mathrm{Ca}^{2+}$ - dependent phospholipid-binding protein has high affinity towards phosphatidylserine and serves as a probe in flow cytometry assay (Donal et al, 2009). Propidium 
Venkatesh et al., Afr J Tradit Complement Altern Med., (2017) 14 (3): 167-174

doi:10.21010/ajtcam.v14i3.18

iodide a dye being impermeable into viable cells can easily penetrate inside dead cells due to the permeability of dead cell membrane. Considering these features, the Annexin V and Propidium iodide were conjugated to use for the early and late apoptotic detection.

The cultured treated and untreated MEG-01 cells were suspended and centrifuged for $5 \mathrm{~min}$ at room temperature. The cell pellet was used according to BD Annexin V: FITC Apoptosis Detection Kit protocol and analyzed the samples using flow cytometry.

HPLC: Compositional analysis of positive fraction obtained after column chromatography was done using a HPLC system. The fraction was subjected to a reversed-phase HPLC on a semi preparative C-18 column, at $25^{\circ} \mathrm{C}$ with the DAD detector at wavelength $210 \mathrm{~nm}$. Two solvent mixtures (solvent A is 100\% methanol and solvent B is 100\% dichloromethane) were used for the elution. The column was equilibrated with solvent mixture in the ratio 1:1. After injecting the sample of $20 \mu \mathrm{l}$ the elution was carried out at a flow rate of $1 \mathrm{ml} / \mathrm{min}$ for about $60 \mathrm{~min}$ (Panawan et al, 2015; Ji-Yeon et al, 2016).

\section{Results}

Thin layer chromatography: TLC was used to separate the crude plant extract into different fractions present if any in the methanolic extract.

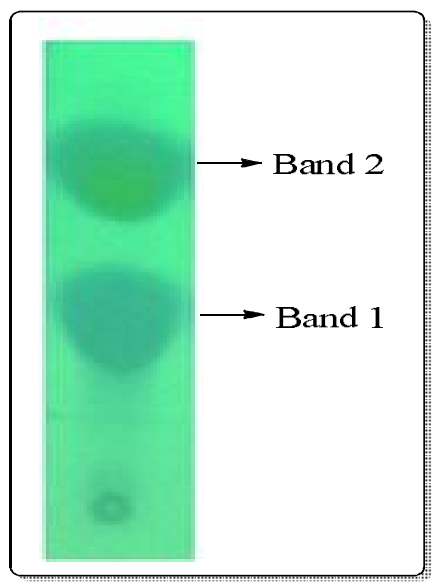

Figure 2: Thin layer chromatogram showing the separation of methanolic extract into two bands under UV light. Thymidine uptake assay: Antiproliferation activity for TLC separated fractions were assayed on MEG01 (human Megakaryoblastic) cell line through thymidine uptake assay.

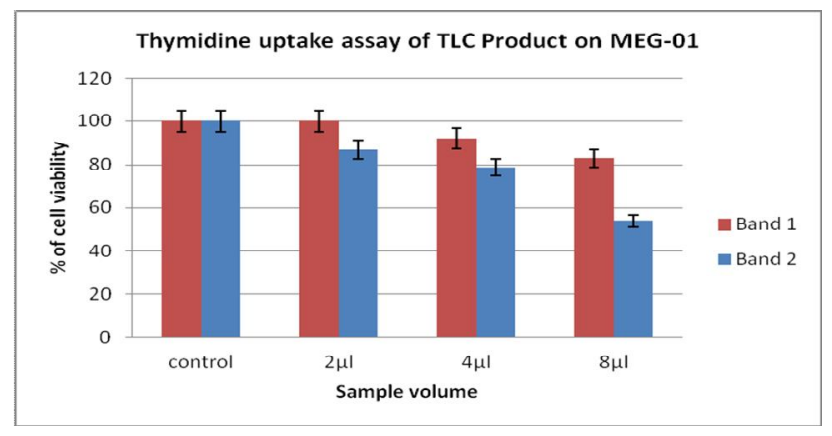

Figure 3: The reduction in cell proliferation of band 1 and band 2 along with increase in quantity has been indicated in the graph. Among two bands, the band 2 showed prominent reduction in cell viability of MEG01.

From the figure 3 it is confirmed that, the band 2 was having more anti-proliferation activity when compare to the band 1.

DNA fragmentation assay: Antiproliferation assay was also in correlation with the DNA degradation assay carried out in our study for the two fractions separated by TLC. The figure 4 indicates that, the lane B2 shows clear fragmentation of the sample indicating the apoptotic activity possessed by band 2 compounds.

$\mathrm{e}$

Figure 4: lane B1 indicates the DNA sample treated with band 1, lane B2 indicates the DNA sample treated with band 2 and lane PC indicates the DNA sample treated with positive control. 
Venkatesh et al., Afr J Tradit Complement Altern Med., (2017) 14 (3): 167-174 doi:10.21010/ajtcam.v14i3.18

Silica gel Column Chromatography: Further dilution and elution of band 2 (obtained from TLC separation) through column chromatography has resulted in five fractions. Approximately eight to ten $\mathrm{ml}$ of elution for each fraction were collected (confirmed through TLC) and used for the study of activity.

Thymidine uptake assay: The fractions from column chromatography were analyzed for antiproliferation activity on MEG01 cells through thymidine uptake assay.

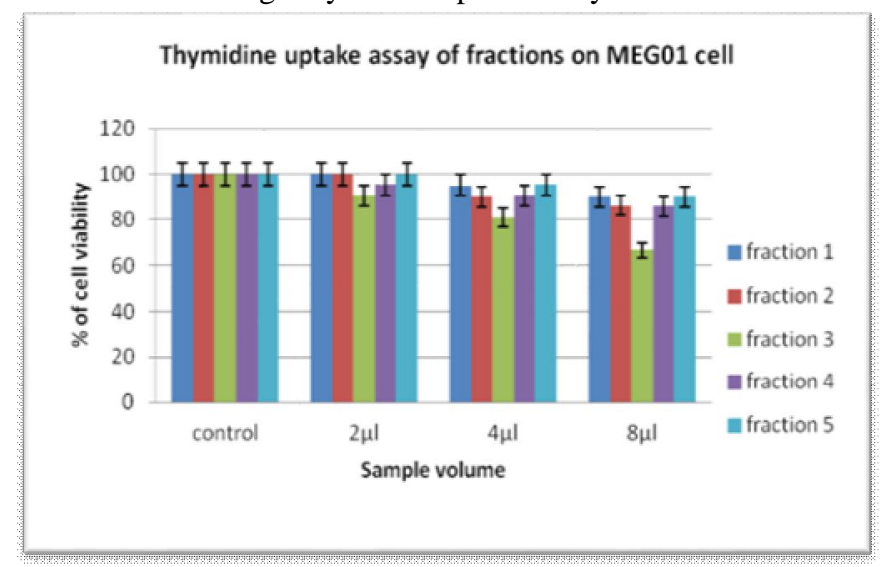

Figure 5: The graph indicates the reduction of MEG01 cell proliferation rate by the column chromatography fractions accordingly with increase in quantity.

Nuclear staining assay: Nuclear staining assay was processed to confirm the apoptotic activity of fraction 3. Nuclear staining of MEG01 cell in negative control shows the viable cells that are permeable only to Acridine orange causing green fluorescence where as in positive control and fraction 3 treated cells shows orange fluorescence due to binding of EtBr to nucleic acids and thereby indicating the apoptotic activity.

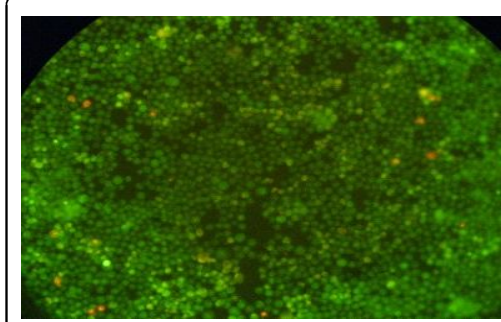

Negative control

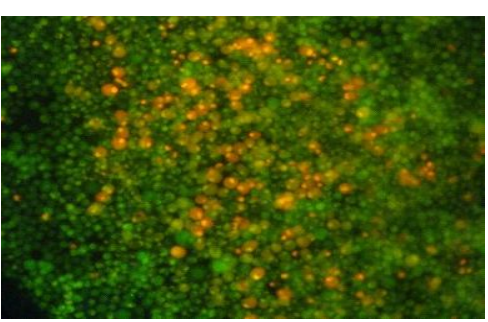

Positive control

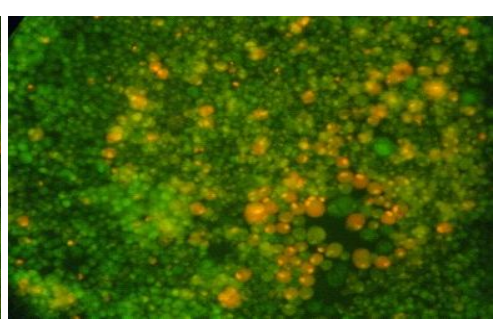

Fraction $3(8 \mathrm{ml})$

Figure 6: Nuclear staining of MEG01 cell with negative, positive control and fraction 3.

Flow cytometry analysis: Flow cytometry analysis for Fraction 3 was also done using BD Annexin V: FITC Apoptosis Detection Kit I (BD bioscience) to estimate the apoptosis activity. The MEG01 cells either untreated (Ctrl) or treated with fraction 3 sample were subsequently stained with Annexin V and Propidium Iodide (PI). The logarithmic amplification of green and red fluorescent signals was measured by flow cytometry. Viable cells (LL) are both Annexin V and PI negative. Early apoptotic cells (LR) bind to Annexin V but not to PI. Late apoptotic (UR) are both Annexin V and PI positive. Some very late apoptotic cells (UL) stain with PI only. 
Venkatesh et al., Afr J Tradit Complement Altern Med., (2017) 14 (3): 167-174

doi:10.21010/ajtcam.v14i3.18
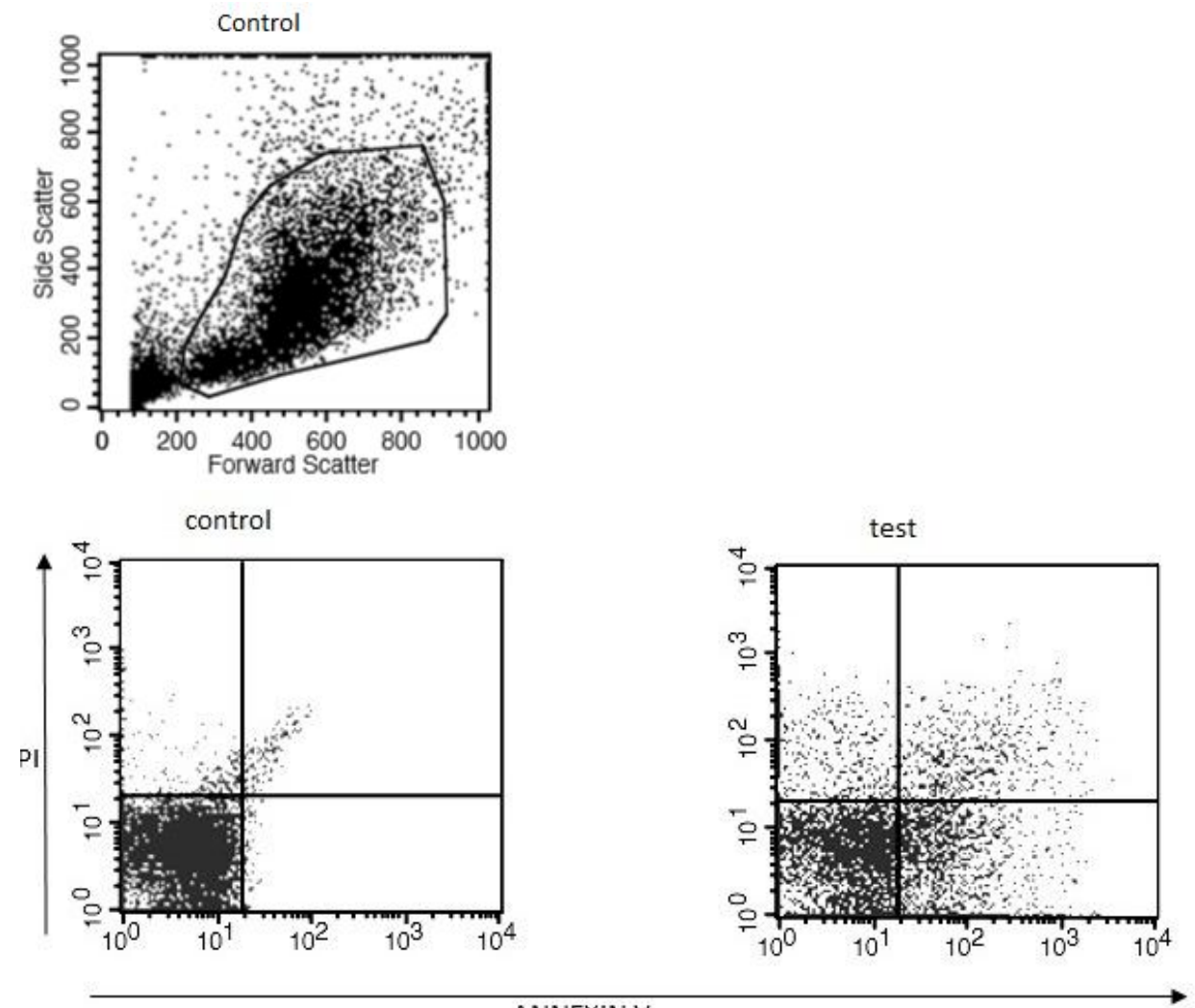

ANNEXIN V

\begin{tabular}{crr} 
Quad & $\%$ Gated & $\%$ Total \\
\hline UL & 2.76 & 1.97 \\
UR & 1.94 & 1.39 \\
LL & 94.29 & 67.39 \\
LR & 1.01 & 0.72
\end{tabular}

\begin{tabular}{crr} 
Quad & $\%$ Gated & $\%$ Total \\
\hline UL & 7.72 & 4.62 \\
UR & 9.33 & 5.58 \\
LL & 57.21 & 34.22 \\
LR & 25.73 & 15.39
\end{tabular}

Figure 7: Detection of apoptotic cells through flow cytometry was done by $\mathrm{Ca}^{2+}$ - dependent phospholipid-binding fluorochrome tagged Annexin V along with Propidium Iodide (PI).

HPLC: The composition analysis of fraction 3 was conducted through reversed-phase HPLC on a semi preparative C18 column.

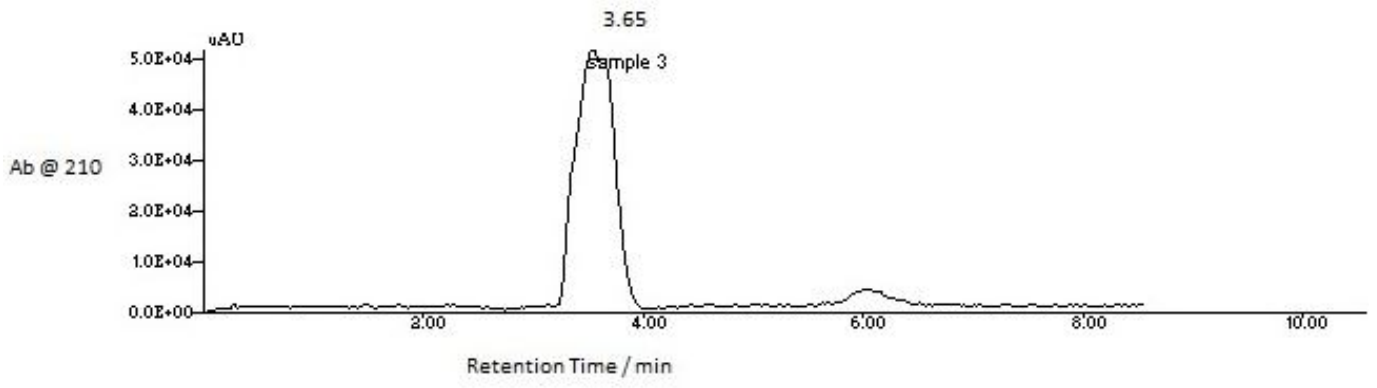

Figure 8: HPLC chromatogram of $3^{\text {rd }}$ fraction, showed a single peak at retention time 3.65 minutes.

\section{Discussion:}

TLC: The initial separation of methanolic extract through TLC (Sasidharan et al. 2011) showed two prominent bands with methanol and dichloromethane solvent mixture taken at the ratio 6:4 whereas, the separation was improper with other solvent mixtures and ratios tried. Among the two bands obtained, the second band still retains certain color mixture which may indicate the presence of different compositions. Before going to the further purification, the characteristic analysis of both the bands was assessed for anti-proliferation and apoptosis activity.

Thymidine uptake assay: The thymidine uptake assay (Giridharan et al. 2002; Belakavadi et al. 2005) to check antiproliferation activity in MEG01 cells was performed twice, first was done on the bands obtained from TLC and 
Venkatesh et al., Afr J Tradit Complement Altern Med., (2017) 14 (3): 167-174 doi:10.21010/ajtcam.v14i3.18

analyzed that the second band showed the reduction in proliferation of MEG01 cells in comparison to the first band indicating that band 2 posses more active compounds in it. Second on the fraction obtained through silica gel column chromatography and in result the fractions 2, 3 and 4 showed the anti-proliferation activity among which the fraction 3 was more prominent. This gave the further lead to concentrate on fraction 3 for analysis.

DNA fragmentation assay: The apoptosis activity of TLC separated bands when checked with the DNA degradation assay (Park et al. 2013) showed that, the band 2 possess the prominent bioactive compound having both antiproliferation as well as the apoptosis activity. This made the band 2 a clear selection for further purification.

Nuclear staining assay: The apoptosis activity was analyzed for fraction 3 in comparison with the activity of 2Amino- $N$-quinolin-8-yl-benzenesulfonamide (Sigma Aldrich), taken as positive control. Both the positive control and fraction 3 produced orange fluorescent with green fluorescent in background due to apoptotic activity indicating that, the ruptured nucleus released apoptotic bodies containing DNA binds to $\mathrm{EtBr}$ and produced orange fluorescent (Srinivas et al, 2003).

Flow cytometry analysis: Further analysis through flow cytometry using $\mathrm{Ca}^{2+}$ dependent phospholipid-binding fluorochrome tagged Annexin V along with Propidium Iodide; the fraction 3 was confirmed that it has more apoptotic activity. In the figure 7 the presence of cells in lower left square indicates the apoptotic cells and this clearly indicates the apoptotic activity by fraction 3 (Donal et al, 2009).

HPLC: The HPLC analysis of fraction 3 produced a single peak at a retention time $3.65 \mathrm{~min}$. The single peak and the early elution indicate the presence of pure and polar compound (Panawan et al, 2015; Ji-Yeon et al, 2016).

\section{Conclusion}

The phytomedicines can be developed as an alternative and are relatively inexpensive than modern drugs (Huang et al, 2006; Neelam et al, 2014). In our current study emphasis is laid on the apoptotic and antileukemic activity of the phytoconstituent where in purification and analytical study of methanolic extract of WT revealed a single peak in HPLC indicating the presence of pure compound with apoptotic and anti-leukemia activities and encouraging for further study on structural analysis.

\section{References}

1. American Cancer Society. Cancer Facts \& Figures 2015. (2015). Atlanta: American Cancer Society.

2. Barrero, A.F., Herrador, M.M., Arteaga, P., Arteaga, J.F., Arteaga, A.F. (2012). Communic acids: occurrence, properties and use as chirons for the synthesis of bioactive compounds. Molecules., 17: 1448 - 1467.

3. Barrero AF, Herrador MM, Arteaga P, Rosas-Romero A, Arteaga JF. (2006). Antioxidant activity of diterpenes and polyphenols from Ophryosporus heptanthus. J Agric Food Chem., 54(7): pp2537 - 2542.

4. Belakavadi, M., Salimath, B.P. (2005). Mechanism of inhibition of ascites tumor growth in mice by curcumin is mediated by NF-kB and caspase activated DNase. Mol Cell Biochem. 273: 57 - 67.

5. Casiglia, S., Bruno, M., Senatore, F. and Rosselli, S. (2016). Chemical composition of essential oils of Anthemis secundiramea Biv. subsp. secundiramea (Asteraceae) collected wild in Sicily and their activity on micro-organisms affecting historical art craft. Natural product research., 4: 1 - 10.

6. Donald, W., Joanna, S. and Zbigniew, D. (2009). Flow cytometry-based apoptosis detection Methods Mol Biol: 559 .

7. Fatma, S.W., Khozirah, S., Johnson, S., Nordin, H.J. L. and Dachriyanus, H. (2016). Cytotoxic Properties and Complete Nuclear Magnetic Resonance Assignment of Isolated Xanthones from the Root of Garcinia cowa Roxb. Pharmacogn Mag.1: s52 - s56.

8. Giridharan, P., Somasundaram, S.T., Perumal, K., Viphwakarama, N.P., Velmurugan, R. (2002). Novel substituted methylenedioxylignan suppresses proliferation of cancer cells by inhibiting telomerase and activation of C-myc and caspases leading to apoptosis. Br J Can. 87: 98 - 105.

9. Huang, X., Ou, S., Tang, S., Fu, L. and Wu, J. (2006). Simultaneous determination of trilobolide-6-Oisobutyrates A and B in Wedelia trilobata by gas chromatography. Chin. J. Chromatogr., 24: 499-502.

10. Ji-Yeon, K., Ohsuk, K., Jin Young, G. and Doo-Byoung, O. (2016). Data for analysis of mannose-6-phosphate glycans labeled with fluorescent tags. Data in Brief. 7: 1531 - 1537.

11. Na, An., Zhongfei, Tao., Saisai, Li., Haiyan, Xing., Kejing, Tang., Zheng, Tian., Qing, Rao., Min, Wang. and Jianxiang, Wang. (2016). Construction of a new anti-CD19 chimeric antigen receptor and the antileukemia function study of the transduced T cells. Oncotarget. 7(9): $10638-10649$.

12. Neelam, B., Titpawan, N. and Teerapol, S*. (2014) Wedelia trilobata L.: A Phytochemical and Pharmacological Review. Chiang Mai J. Sci., 41(3): 590-605.

13. Panawan, S., Watcharapong, C., Sugunya, M., Suwaporn, L., Somsuda, T. and Vijittra, L. (2015). Structures of Phytosterols and Triterpenoids with Potential Anti-Cancer Activity in Bran of Black Non-Glutinous Rice Nutrients. Nutrients. 7: 1672 - 1687. 
Venkatesh et al., Afr J Tradit Complement Altern Med., (2017) 14 (3): 167-174

doi:10.21010/ajtcam.v14i3.18

14. Park, H.S., Hwang, H.J., Kim, G.Y., Cha, H.J. and Kim, W.J. (2013). Induction of apoptosis by fucoidan in human leukemia U937 cells through activation of p38 MAPK and modulation of Bcl-2 family. Mar Drugs. 11(7): 2347 2364.

15. Sasidharan, S.,Chen, Y.,Saravanan, D.,Sundram, K. M.,Yoga Latha, L. (2011). Extraction, Isolation and Characterization of bioactive compounds from plants' extracts. Afr J Tradit Complement Altern Med. 8(1): 1 - 10.

16. Srinivas, G., Anto, R.J., Srinivas, P., Vidhya, L.S. and Priya, S.V. (2003). Emodin induces apoptosis of human cervical cancer cells through poly (ADP-ribose) polymerase cleavage and activation of caspase-9. Evr J Pharmaco. 473: $117-125$.

17. Uday, V., Shiva Prasad, K., Chethan, J., Shankar, J. and Satish Kumar, M. (2016). Methanolic Extract of Wedelia trilobata in Antiproliferation and Apoptotic Activity. Natural Products Chemistry \& Research. 4: 210.

18. Veeramuthu, D., Muniappan, A. and Savarimuthu, I. (2006). Antimicrobial activity of some ethnomedicinal plants used by Paliyar tribe from Tamil Nadu, India. BMC Complementary and Alternative Medicine., 6: 35. 